\title{
Socioeconomic correlates of SARS-CoV-2 and influenza H1N1 outbreaks
}

\author{
To the Editor:
}

The severe acute respiratory syndrome coronavirus 2 (SARS-CoV-2) outbreak has disrupted social and economic life over large parts of the world [1]. The countermeasures designed and enforced by organisations and governments in order to contain the pandemic have had debated impact on its spread, but especially on societal structure and economic output [2]. In a fashion only precedented by war, an infectious pandemic challenges vulnerabilities of our societal structure, economic activity and healthcare [3]. While the battle for the discovery of the disease's origins, pathogenesis and cure is ongoing, a deeper understanding of its pattern of spread is sought [4]. Geographic patterns of disease burden are essential in understanding our societal and biological vulnerabilities to disease, as well as the progress of our worldwide battle against disease [5]. To this end, the way the SARS-CoV-2 pandemic has affected different countries could provide valuable clues to the nature of the disease, as well as our societal and economic weaknesses that propagate it.

For this, the socioeconomic correlates of the SARS-CoV 2 outbreak by country were analysed. Cumulative confirmed SARS-CoV 2 cases and deaths by country were recorded on 11 April 2020, when the pandemic had caused 1614949 cases and 99886 deaths [6]. Data from the 2009-2010 H1N1 influenza outbreak, the only comparable recent epidemic, were used as control [7]. Viral outbreak data by country were cross-examined with socioeconomic indices (all accessed on 11 April 2020), including: population, urban population (persons and $\%$ of total), median population age (years), and population density (persons per $\mathrm{km}^{2}$ ) [8]; inflation-adjusted health expenditure (total in USD, and expenditure in USD per person) (2015 World Health Organization (WHO) data adjusted for 2011 purchasing power parity) [9]; and annual gross domestic product (GDP; USD per person and total in USD) [10].

To prevent testing bias, only 21 countries that reported more than 1500 cases and more than 100 deaths for both pandemics were selected for analyses, which included (in geographical order by WHO region) the USA, Canada, Mexico, Peru, Brazil and Equador (Americas); the UK, Germany, France, Poland, Czech Republic, Portugal, Spain, Italy, Romania and Turkey (Europe); Egypt and Iran (Eastern Mediterranean); China and South Korea (Western Pacific); and India (South-East Asia). Decimal logarithmic transformation of the raw data and exclusion of Iran rendered all variables normally distributed (Kolmogorov-Smirnov test) and yielded data from 20 countries amenable to parametric analyses. In total, 42 correlations were done, and the probability threshold of statistical significance $(\mathrm{p}=0.05)$ was reset by Bonferroni correction to $\mathrm{p}=0.05 / 42=1.2 \times 10^{-3}$, revealing 17 significant correlations, for which linear regressions were performed. All analyses and graphs were done using Excel (Microsoft, Redmond, WA, USA) and Prism v.8.0. (GraphPad, San Diego, CA, USA). Interestingly, and in accord with other lines of evidence [11], the SARS-CoV-2 and H1N1 influenza outbreaks were fundamentally different in affecting the 20 countries examined: the confirmed cases and deaths caused by the two outbreaks were not correlated. Surprisingly, SARS-CoV-2 cases were strongly correlated with economic output as measured by GDP and health expenditure, in stark contrast with H1N1 influenza cases, which were not associated with the above or any other socioeconomic index. SARS-CoV-2 deaths were correlated with SARS-CoV-2 cases underpinning the lethality of the disease, something not evident with H1N1 deaths, which were correlated with total and urban population size, GDP and health expenditure.

To confirm these results, confirmed SARS-CoV-2 cases and deaths by country were recorded again on 16 April 2020, when 1918138 individuals were affected and 123126 had died, and on 12 May 2020, when cumulative numbers had risen to 4098017 confirmed cases and 283272 deaths [6]. Using the criteria described above,

@ERSpublications

Geographic disease patterns of the SARS-CoV-2 and the H1N1 influenza pandemics are crossexamined with socioeconomic indices, revealing that the two outbreaks are fundamentally different and that SARS-CoV-2 spread is linked with economic output https://bit.ly/3fqkiyp

Cite this article as: Kaiser JC, Stathopoulos GT. Socioeconomic correlates of SARS-CoV-2 and influenza H1N1 outbreaks. Eur Respir J 2020; 56: 2001400 [https://doi.org/10.1183/13993003.01400-2020]. 

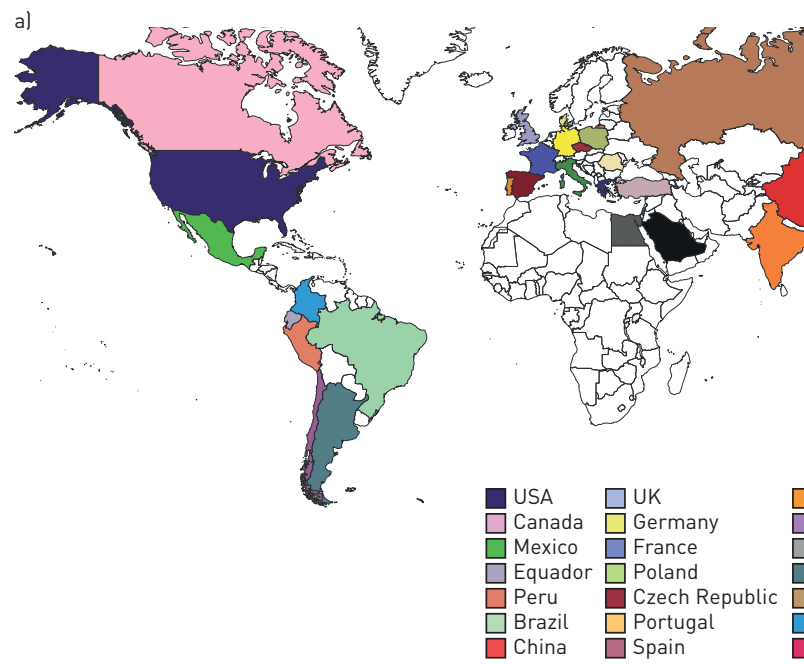

b)

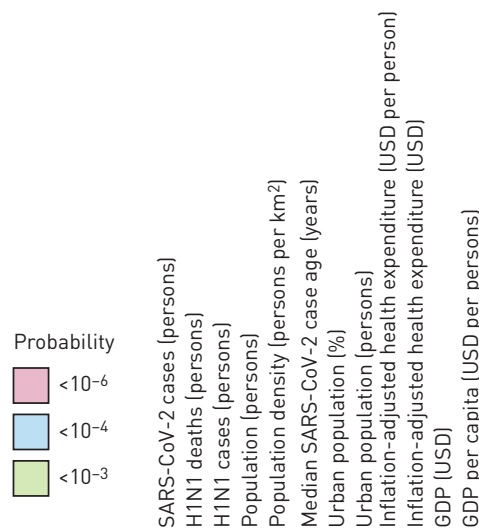

SARS-CoV-2 deaths (persons) ARS-CoV-2 cases (persons) H1N1 deaths (persons) H1N1 cases (persons)
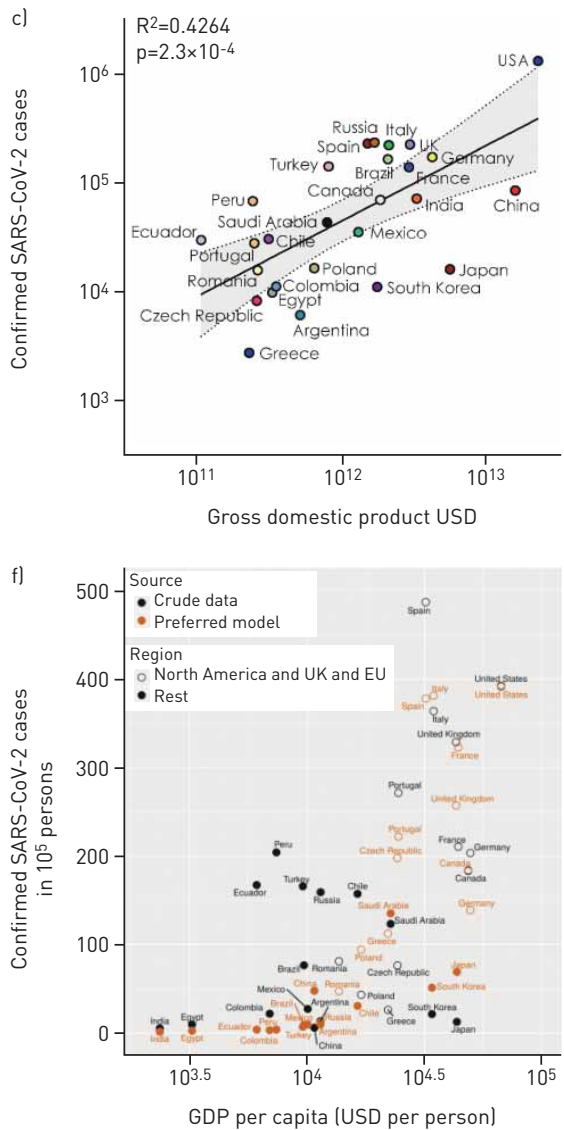
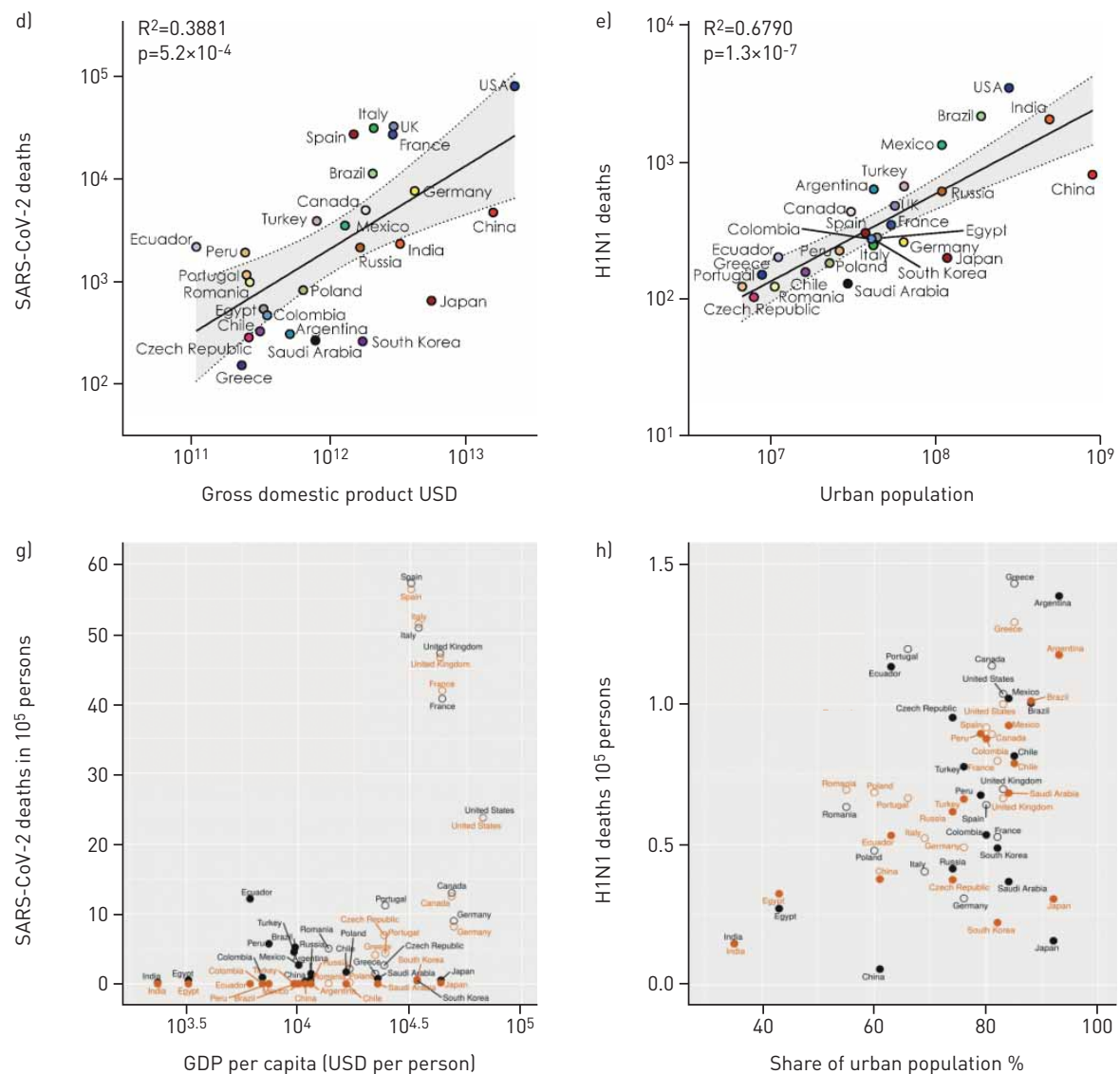

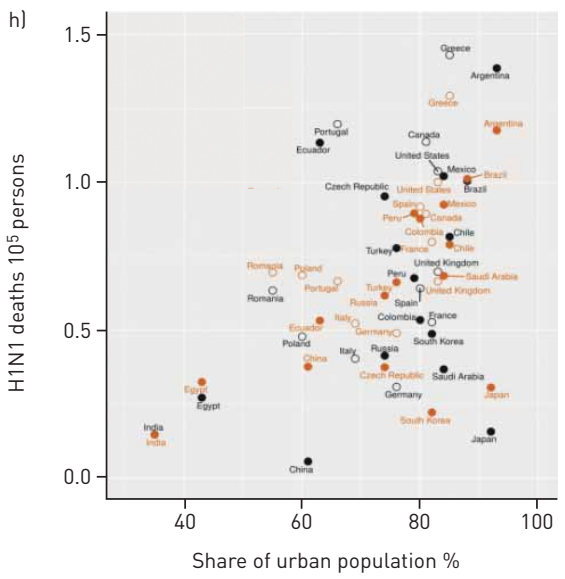

FIGURE 1 Severe acute respiratory syndrome coronavirus 2 (SARS-CoV-2) and H1N1 influenza pandemics in 27 countries. a) Colour-coded map and legend of the 27 countries analysed on 12 May 2020. b) Heatmap of the correlations observed on 12 May 2020. c-e) Significant bivariate correlations of the SARS-CoV 2 and H1N1 outbreaks with socioeconomic indices. Shown are color-coded raw data points, Pearson's correlation coefficients $\left(R^{2}\right)$ and probability values ( $p$ ), and linear regression lines with 95\% confidence intervals (grey zones). GDP: annual gross domestic product. $f-h)$ Crude rates for population-adjusted SARS-CoV-2 deaths and cases and H1N1 deaths in comparison with country-specific rates expected by the preferred general linear model.

25 and 27 countries, respectively, were eligible for analyses, including the 20 initial countries plus Argentina, Colombia, the Russian Federation, Greece, and Japan on 16 April 2020, as well as Chile and Saudi Arabia on 12 May 2020. Using the analyses detailed above, the results were identical with those of the 11 April 2020 standing. The 27 countries analysed on 12 May 2020 are shown in figure 1a, the bivariate correlation results from this date are shown in figure $1 \mathrm{~b}$, and selected bivariate correlations in figures 1c-e. 
To substantiate the bivariate correlation analysis, generalised linear models (GLMs) were set up to provide a more comprehensive view on the socioeconomic impact. The latest dataset with 27 countries was investigated to ensure the largest possible degree of freedom. Confounding by size effects was avoided by applying the population-adjusted covariables median age, population density, share of urban population, health expenditure per capita and GDP per capita. Regression was performed on population-adjusted SARS-CoV-2 deaths, confirmed SARS-CoV-2 cases and H1N1 cases with a Gaussian GLM using a log link function. The preferred model was chosen by goodness-of-fit measured by the Akaike information criterion (AIC). Regression on SARS-CoV-2 deaths guided model development. Deaths are deemed more reliable to assess health outcome compared to cases which are strongly dependent on testing strategies. The preferred model for deaths applied strong linear-quadratic dependences on health expenditure and GDP $(p<0.01)$ whereas the remaining covariables maintained simple linear dependences. The AIC decreased markedly after adding a categorical risk factor pertaining to the group of rich countries from North America, the European Union, or the UK. Applying the preferred model for SARS-CoV-2 deaths to cases yielded only GDP as significant. For H1N1 deaths only the share of urban population remained statistically significant among all covariables (figures $1 \mathrm{f}-\mathrm{h}$ ). All raw data and analysis results are available upon request to the corresponding author in ${ }^{\star}$.xlsx and ${ }^{\star}$.pzfx formats and as $\mathrm{R}^{\star}$ scripts.

The overall view of these findings confirms the results of the bivariate analysis. The preferred model clearly identifies a strong (linear-quadratic) relationship between SARS-CoV-2 to both health expenditure and GDP in rich countries from Europe and North America notably with a GDP per capita $>30000$ USD. On the other hand, for $\mathrm{H} 1 \mathrm{N1}$, urbanisation and possibly population density are the defining socioeconomic risk factors. Interestingly, median population age $(\mathrm{p}=0.7)$ did not emerge as a strong risk factor in the preferred model, although SARS-CoV-2 mortality dramatically increases in the older population. Surprisingly, our dataset of a limited number of socioeconomic covariables explains SARS-CoV-2 mortality remarkably well, especially for countries with GDP per capita >30000 USD. In general, our findings rather point to an unexplained gap between rich countries from both sides of the Atlantic compared to the rest of the world in view of the socioeconomic susceptibility to SARS-CoV-2.

Although the analyses provided here have limitations, including their descriptive nature, the uncertain future course of the current outbreak, and country-dependent reporting biases, they suggest an ugly truth: namely that the current SARS-CoV 2 pandemic may actually target economic growth. Pending further validation, this fact is alarming, since it implies that we have to change the ways economic output is achieved in the future. The results are plausible, since modern economic growth involves mass farming and deforestation, practices which are known to decrease inter-species distance and to facilitate inter-species infectious transmission [12]. Modern economy also requires mass travel and large working communities, which facilitate human-to-human transmission of viral outbreaks [4].

The comparison between the two pandemics undertaken here is interesting, since they were fundamentally different. No lockdown measures were enforced a decade ago against the H1N1 influenza, when antiviral agents were available and a vaccine was offered rather shortly after the outbreak. In contrast, the current SARS-CoV 2 pandemic has flooded hospitals and has caused so far an alarming number of deaths. So indeed, current measures taken now have been precedented only by war and the Spanish flu pandemic in the early 20th century, with which a financial comparison is impossible as lifestyle has dramatically shifted since then. The authors are unsure whether the observed association of the SARS-CoV 2 outbreak with economic output is due to the pandemic itself, the countermeasures taken against it, or the direct and indirect costs of the disease. To this end, the importance of the financial and social lockdowns cannot be quantitatively estimated by the methodology used here and remains subject to further investigation.

In conclusion, it is proposed that the incipient and much anticipated restart of economies after the SARS-CoV-2 pandemic has to be based on the lessons learnt. In this regard, ways employed to achieve economic growth in a fashion that prevents future infectious outbreaks should be considered. In addition, the impact of the lockdown measures imposed on society and production have to be carefully evaluated after the current pandemic has hopefully subsided.

Jan Christian Kaiser ${ }^{1}$ and Georgios T. Stathopoulos $\oplus^{2}$

${ }^{1}$ Institute of Radiation Medicine (IRM), Helmholtz Center Munich for Environmental Health (Helmholtz Zentrum München für Gesundheit und Umwelt, HMGU), Oberschleissheim, Germany. ${ }^{2}$ Comprehensive Pneumology Center (CPC) and Institute for Lung Biology and Disease (iLBD), Helmholtz Center Munich for Environmental Health (Helmholtz Zentrum München für Gesundheit und Umwelt, HMGU); and German Center for Lung Research (Deutsches Zentrum für Lungenforschung, DZL), Neuherberg, Germany.

Correspondence: Georgios T. Stathopoulos, Comprehensive Pneumology Center, Helmholtz Zentrum München; MaxLebsche-Platz 31, 81377 Munich, Germany. E-mail: stathopoulos@helmholtz-muenchen.de

Received: 25 April 2020 | Accepted after revision: 4 July 2020 
Acknowledgements: The authors extend their warm thanks to the World Health Organization for the creation and maintenance of the coronavirus disease (COVID-19) situation dashboard (https://who.sprinklr.com/) that made this study possible, as well as the editors and reviewers of the European Respiratory Journal for their constructive comments on this report. G.T. Stathopoulos thanks his dear friend Theodoros Belezonis for insightful discussions that prompted this investigation.

Data availability: All raw data and analysis results are available upon request to the corresponding author in ${ }^{*}$.xlsx and ${ }^{*}$.pzfx formats and as $\mathrm{R}^{*}$ scripts.

Author contributions: J.C. Kaiser provided the multivariate data analysis and modelling. G.T. Stathopoulos collected and descriptively analysed the data, is the guarantor of the study's integrity, wrote the manuscript, and designed the final version of the figure.

Conflict of interest: J.C. Kaiser has nothing to disclose. G.T. Stathopoulos has nothing to disclose.

\section{References}

1 Munster VJ, Koopmans M, van Doremalen N, et al. A novel coronavirus emerging in China - key questions for impact assessment. N Engl J Med 2020; 382: 692-694.

2 Parmet WE, Sinha MS. Covid-19 - the law and limits of quarantine. N Engl J Med 2020; 382: e28.

3 Studdert DM, Hall MA. Disease control, civil liberties, and mass testing - calibrating restrictions during the Covid-19 pandemic. N Engl J Med 2020; 383: 102-104.

4 Kraemer MUG, Yang CH, Gutierrez B, et al. The effect of human mobility and control measures on the COVID-19 epidemic in China. Science 2020; 368: 493-497.

5 GBD 2016 Disease and Injury Incidence and Prevalence Collaborators. Global, regional, and national incidence, prevalence, and years lived with disability for 328 diseases and injuries for 195 countries, 1990-2016: a systematic analysis for the Global Burden of Disease Study 2016. Lancet 2017; 390: 1211-1259.

6 World Health Organization. Coronavirus Disease (COVID-19) Situation Dashboard. Date last updated: 21 August 2020. Dates accessed: 11 April 2020, 16 April 2020, and 12 May 2020. https://who.sprinklr.com/.

7 Wikipedia. 2009 Swine Flu Pandemic By Country. https://en.wikipedia.org/wiki/2009_swine_flu_pandemic_by_ country Date last updated: 17 April 2020. Date last accessed: 25 April 2020.

8 United Nations, Department of Economic and Social Affairs, Population Division (2019). World Population Prospects 2019, Volume I: Comprehensive Tables (ST/ESA/SER.A/426). https://population.un.org/wpp/ Publications/Files/WPP2019_Volume-I_Comprehensive-Tables.pdf Date last updated: 17 April 2019. Date last accessed: 25 April 2020.

9 World Health Organization. Global Health Expenditure Database. https:/apps.who.int/nha/database/Select/ Indicators/en Date last updated: 25 April 2019. Date last accessed: 25 April 2020.

10 International Monetary Fund. World Economic Outlook Database, April 2019. www.imf.org/external/pubs/ft/weo/ 2019/01/weodata/download.aspx Date last updated: 25 April 2019. Date last accessed: 25 April 2020.

11 Tang X, Du R, Wang R, et al. Comparison of hospitalized patients with ARDS caused by COVID-19 and H1N1. Chest 2020; 158: 195-205.

12 Shinde V, Bridges CB, Uyeki TM, et al. Triple-reassortant swine influenza A (H1) in humans in the United States, 2005-2009. N Engl J Med 2009; 360: 2616-2625. 